\title{
Anomalous Cosmic Rays: The Principal Source of High Energy Heavy Ions in the Radiation Belts
}

\author{
R.A. Mewaldt, R.S. Selesnick and J.R. Cummings \\ California Institute of Technology, Pasadena, California
}

\begin{abstract}
Recent observations from SAMPEX have shown that "anomalous cosmic rays" are the principal source of high energy $(>10 \mathrm{MeV} /$ nuc) heavy ions trapped in the radiation belts. This component of interplanetary particles is known to originate from interstellar atoms that has been accelerated to high energies in the outer heliosphere. The mechanism by which anomalous cosmic rays with $\sim 1$ to $\sim$ $50 \mathrm{MeV} /$ nuc are trapped in a radiation belt at $L \approx 2$ has now been verified. We discuss models for accelerating and trapping anomalous cosmic rays and review observations of their composition, energy spectra, pitch angle distribution, and time variations. Extrapolation of the fluxes observed at $\sim 600 \mathrm{~km}$ to higher altitude and other time periods is also discussed.
\end{abstract}

\section{INTRODUCTION}

Just over twenty years ago a new component of cosmic rays was discovered when unexpected enhancements were observed in the energy spectra of 1 to $50 \mathrm{MeV} /$ nuc $\mathrm{He}, \mathrm{N}$, and $\mathrm{O}$ measured in interplanetary space during solar quiet times. Soon after the discovery of this so-called "anomalous cosmic ray" (ACR) component, Fisk, Koslovsky, and Ramaty [1974] proposed what has proven to be the correct explanation for its origin, suggesting that ACRs represent a sample of neutral interstellar particles that have drifted into the heliosphere, become ionized by the solar wind or UV radiation, and then accelerated to energies of tens of $\mathrm{MeV} /$ nuc in the outer heliosphere. Once accelerated, they can re-enter the inner heliosphere as low energy cosmic rays (see Figure 1). There is now evidence for ACR contributions to seven elements $(H$, $\mathrm{He}, \mathrm{C}, \mathrm{N}, \mathrm{O}, \mathrm{Ne}$, and $\mathrm{Ar}$ ) and over the past two decades the properties of ACRs have been studied extensively throughout the heliosphere (see reviews in Simpson [1995] and Klecker [1995]).

Shortly after the discovery of ACRs Blake and Friesen [1977] suggested a mechanism for trapping ACRs in the magnetosphere, but it was more than a decade later that the first

Radiation Belts: Models and Standards

Geophysical Monograph 97

Copyright 1996 by the American Geophysical Union solid evidence for this mechanism was provided [Grigorov et al., 1991]. With the launch of the Solar, Anomalous, and Magnetospheric Particle Explorer (SAMPEX) in 1992 it became possible to study high-energy heavy ions in the magnetosphere in detail for the first time. SAMPEX measurements have shown that anomalous cosmic rays are the dominant source of heavy ions with $>10 \mathrm{MeV} /$ nuc in the magnetosphere, and that they are located in a relatively narrow belt at $L \approx 2$ [Cummings et al., 1993, 1994]. In this paper we review briefly the properties of anomalous cosmic rays in interplanetary space, describe models for their acceleration and subsequent trapping in the magnetosphere, and present observations of trapped ACRs provided by SAMPEX over the past three years.

\section{ANOMALOUS COSMIC RAYS IN INTERPLANETARY SPACE}

The model by Fisk, Koslovsky and Ramaty (hereinafter FK\&R) predicts that there should be ACR contributions to species that are mainly or partially neutral in the interstellar medium. The observed abundances of $\mathrm{He}, \mathrm{C}, \mathrm{N}, \mathrm{O}, \mathrm{Ne}$, and Ar are generally consistent with this picture, and provide a means of measuring the composition of the neutral interstellar medium [Cummings and Stone, 1995]. For example, the low abundance of carbon in ACRs (see Figure 1) implies that only $\sim 1 \%$ of the carbon in the interstellar medium is in a neutral state. The model of FK\&R also predicts that ACRs should be singly charged, in contrast to galactic cosmic rays, which are essentially fully stripped, and there is now abundant evid- 


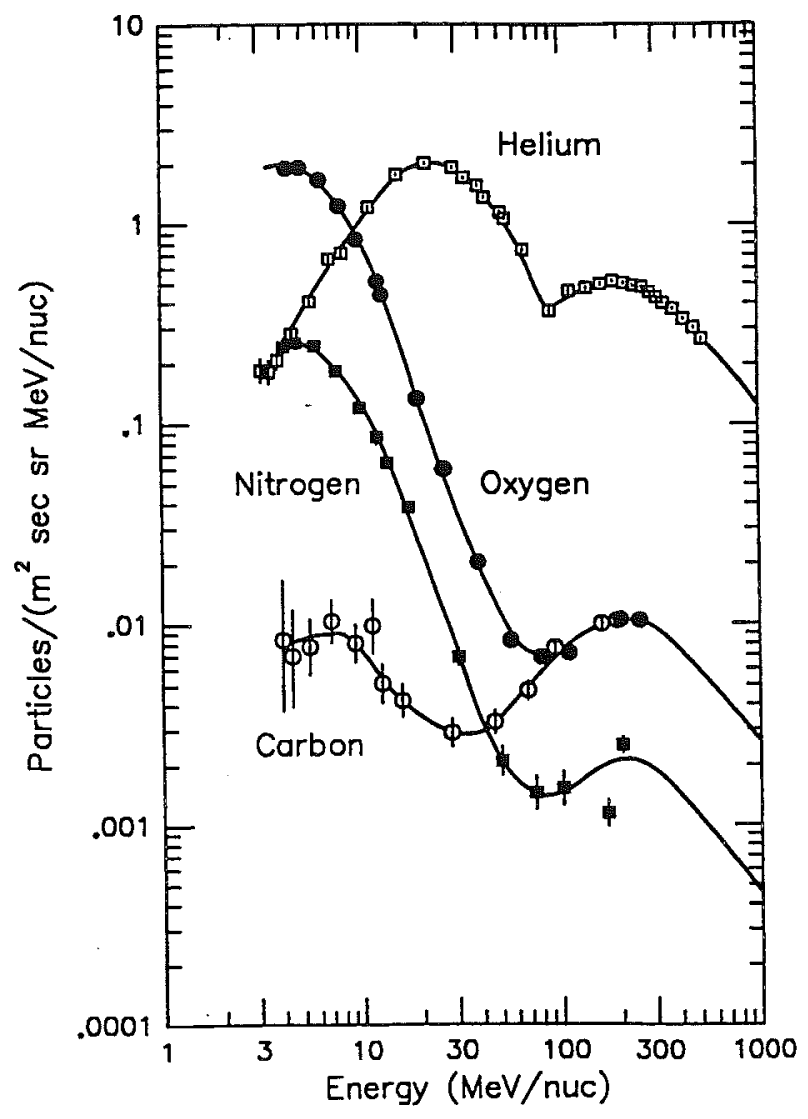

Figure 1. Energy spectra of $\mathrm{He}, \mathrm{C}, \mathrm{N}$, and $\mathrm{O}$ observed by Voyager 2 at $\sim 23 \mathrm{AU}$ during the 1987 solar minimum illustrating the anomalous low-energy enhancements in the spectra (from Mewaldt et al. [1994]). At energies $>50 \mathrm{MeV} /$ nuc galactic cosmic rays dominate.

ence that the bulk of ACRs with $\sim 10 \mathrm{MeV} /$ nuc are singly charged (see Klecker et al. [1995] and references therein).

As Pioneer $10 \& 11$, and later Voyager $1 \& 2$, began to explore the outer solar system they found that the intensity of ACRs increased with distance from the Sun, and the distribution of ACRs in the heliosphere has now been measured out to $60 \mathrm{AU}$, and to latitudes as high as $80^{\circ}$ (e.g., Cummings et al. [1995]). Ulysses has recently measured the abundances of the "pick-up" ions that are the seed population for ACR acceleration (e.g., Geiss et al. [1995]). It is now believed that the bulk of ACR acceleration takes place at the solar wind termination shock [Pesses et al., 1981; Jokipii, 1990] estimated to be at a distance of $\sim 80$ to $100 \mathrm{AU}$ from the Sun. Because the access of low energy cosmic rays to inner solar system is strongly affected by interplanetary conditions ("solar modulation"), ACRs are detectable at 1 AU only near solar minimum. Figure 2 illustrates variations in the intensity of 8 to $27 \mathrm{MeV} /$ nuc oxygen over the past 27 years. Note that the intensity at $1 \mathrm{AU}$ varies by a factor $>100$, and is reasonably well represented by the neutron monitor count rate taken to the 25th power.

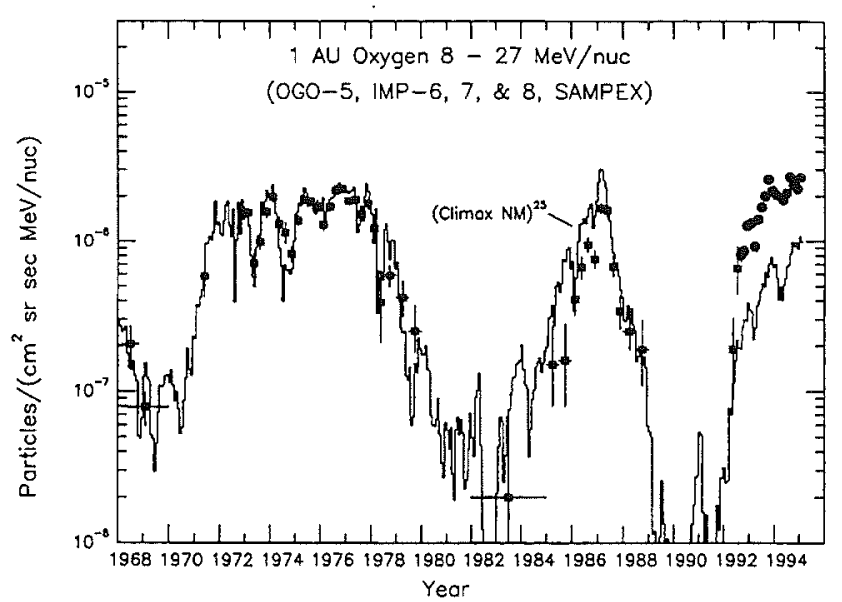

Figure 2. Quiet time measurements in the flux of 8 to 27 $\mathrm{MeV} /$ nucleon oxygen over the past 27 years. Data from 1972 to mid-1992 are from IMP-8, after which they are from the HILT sensor on SAMPEX (B. Klecker, private communication). For references to the earlier data see Mewaldt et al. [1993]. The solid curve is proportional to the Climax neutron monitor counting rate ( $R$. Pyle and $J$. Simpson, private communication) taken to the $25^{\text {th }}$ power.

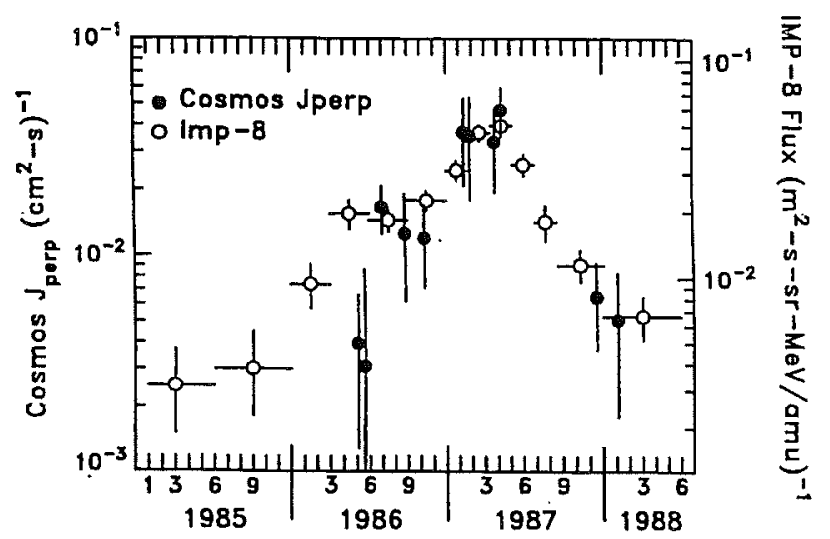

Figure 3. Measurements of the flux of 5 to $30 \mathrm{MeV} /$ nuc trapped oxygen from a series of Cosmos flights at a typical altitude of $\sim 300 \mathrm{~km}$ are compared with the interplanetary flux of 8 to $27 \mathrm{MeV} /$ nuc oxygen measured on IMP-8, and with the scaled Mt. Washington neutron monitor count rate taken to the $30^{\text {th }}$ power (from Grigorov et al. [1991]).

\section{TRAPPING ANOMALOUS COSMIC RAYS}

Long before there was much solid evidence in favor of the model by FK\&R, Blake and Friesen [1977] suggested that if ACRs were indeed singly charged, then some of them would become trapped in the Earth's radiation belts. They reasoned that singly-charged ACRs can penetrate to lower geomagnetic latitude than fully-stripped galactic cosmic rays or solar particles with the same energy/nuc, because they have greater rigidity. If one of these ions were to brush against 


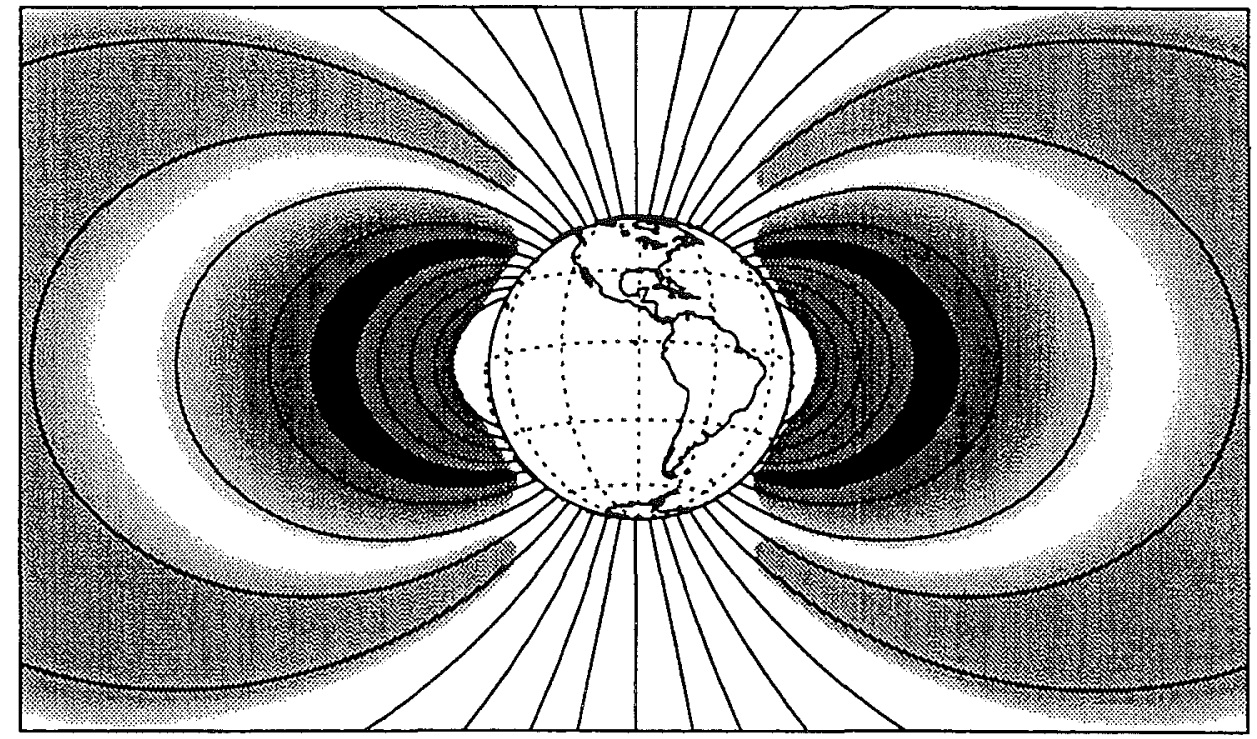

Figure 4. Illustration of the Earth's radiation belts. The narrow belt at $L=2$ (dark shading) includes trapped anomalous cosmic rays that originate from the local interstellar medium.

Table 1. Anomalous Cosmic Ray Abundance Ratios

\begin{tabular}{lcrr}
\hline & \multicolumn{2}{c}{ SAMPEX (1992-1993) } & Voyager 2, 1987 (1) \\
\cline { 2 - 4 } & $\begin{array}{c}\text { Trapped (1) } \\
\text { 16-45 MeV/nucleon }\end{array}$ & $\begin{array}{c}\text { Interplanetary (2) } \\
>17 \mathrm{MeV} / \text { nucleon }\end{array}$ & $\begin{array}{c}\text { Interplanetary } \\
16-30 \mathrm{MeV} / \mathrm{nucleon}\end{array}$ \\
\hline $\mathrm{C} / \mathrm{O}$ & $\sim 0.0004$ & $0.014 \pm 0.009$ & $0.020 \pm 0.004$ \\
$\mathrm{~N} / \mathrm{O}$ & $0.09 \pm 0.01$ & $0.19 \pm 0.03$ & $0.194 \pm 0.013$ \\
$\mathrm{Ne} / \mathrm{O}$ & $0.04 \pm 0.01^{*}$ & $0.06 \pm 0.02^{*}$ & $0.048 \pm 0.006$ \\
\hline
\end{tabular}

* Lower energy limit for SAMPEX $\mathrm{Ne} / \mathrm{O}$ is $18 \mathrm{MeV} /$ nucleon.

(1) Selesnick et al. [1995a]

(2) Mewaldt et al. [1996]

the upper atmosphere, losing some or all of its remaining electrons, its rigidity would suddenly decrease by a large factor (up to eight in the case of singly-charged oxygen). Depending on its pitch angle, it might then become trapped in a stable orbit. Blake [1990] estimated that trapped ACRs might have lifetimes of weeks to months before losing their energy to the residual atmosphere.

While Blake and Friesen had been stimulated by observations from Skylab [Chan and Price, 1975; Biswas et al. 1975], the composition reported by these experiments did not fully agree with that of interplanetary ACRs, and this model received little attention for more than a decade. Grigorov et al. [1991], using dielectric track detectors flown on a series of low-altitude Cosmos flights, observed a population of 5 to $30 \mathrm{MeV} / \mathrm{nuc}$ heavy ions whose angular distribution indicated a trapped population, and whose composition and time variations (see Figure 3) were consistent with that of interplanetary ACRs observed simultaneously by IMP-8. They correctly identified these as the trapped ACRs predicted by Blake and Friesen [1977].

\section{SAMPEX OBSERVATIONS}

Soon after this discovery, SAMPEX was launched into an $82^{\circ}$ inclination low-Earth orbit carrying four instruments (MAST, PET, LICA, and HILT) designed to measure energetic nuclei and electrons over three decades in energy (see Baker et al. [1993] and associated articles in the same issue). Measurements with the MAST instrument on SAMPEX [Cook et al., 1993] quickly showed that trapped ACRs with $>15 \mathrm{MeV} /$ nuc are located in a narrow belt centered at $L \approx 2$ and embedded within the inner Van Allen belt [Cummings et al., 1993ab], as illustrated in Figure 4. Since 1992 SAMPEX has been mapping the distribution and time variations of trapped ACRs, as well as other energetic particle components. These observations have generally confirmed the model 


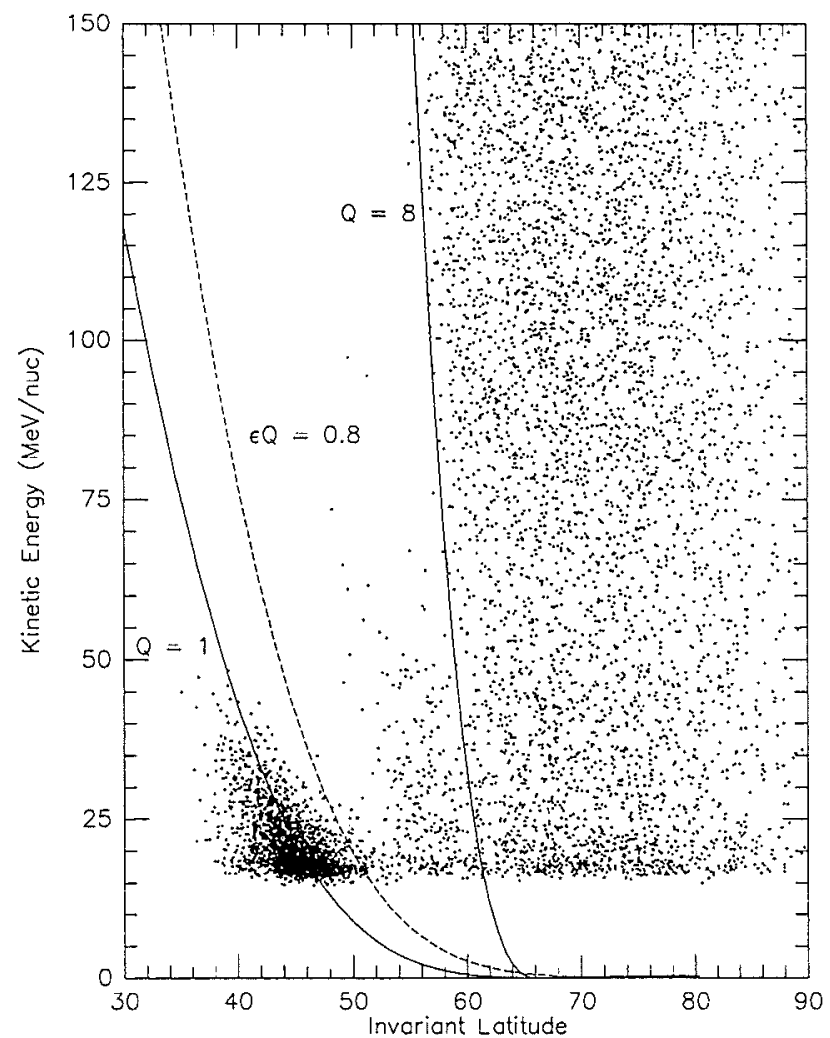

Figure 5. Measured energy/nucleon vs. invariant latitude for oxygen ions observed during solar-quiet days from $7 / 92$ to $2 / 94$. Calculated geomagnetic cutoffs for particles arriving from the west (estimated by Störmer dipole approximation) are shown for singly-ionized $(Q=+1)$ and fully stripped oxygen $(Q=+8)$. At latitudes $\Lambda>60^{\circ}$ a mixture of ACRs and GCRs is observed. At midlatitudes, GCRs no longer have access, but singly-charged ACRs have access. At $\Lambda \approx 45^{\circ}$ a population of trapped ACRs is observed. The dotted line labeled $\varepsilon Q=0.8$ approximately bounds the trapped fluxes (see discussion in text).

of Blake and Friesen [1977], although they have modified a number of details in the model.

To illustrate the distribution of ACRs observed over the SAMPEX orbit Figure 5 shows measured kinetic energy vs. invariant latitude $(\Lambda)$ for oxygen nuclei with $>15 \mathrm{MeV} /$ nuc. For comparison, nominal geomagnetic cutoffs for particles arriving from the west, estimated from the Störmer dipole approximation, are shown for singly ionized and fully stripped oxygen. Three distinct particle populations are evident. At high latitudes $\left(>60^{\circ}\right)$ there is a mixture of GCR and ACR oxygen. At mid-latitudes $\left(\sim 50^{\circ}\right.$ to $\left.60^{\circ}\right)$, fully stripped GCRs are not allowed, but singly-charged (and possibly also multiply-charged) ACRs can be observed down to the appropriate geomagnetic cutoff. At low latitudes $\left(<50^{\circ}\right)$, there is a grouping near and below the estimated cutoff for singlycharged oxygen. Figure 5 demonstrates the use of the Earth's field as a magnetic spectrometer for separating out anomalous cosmic rays from fully stripped GCRs, and this technique

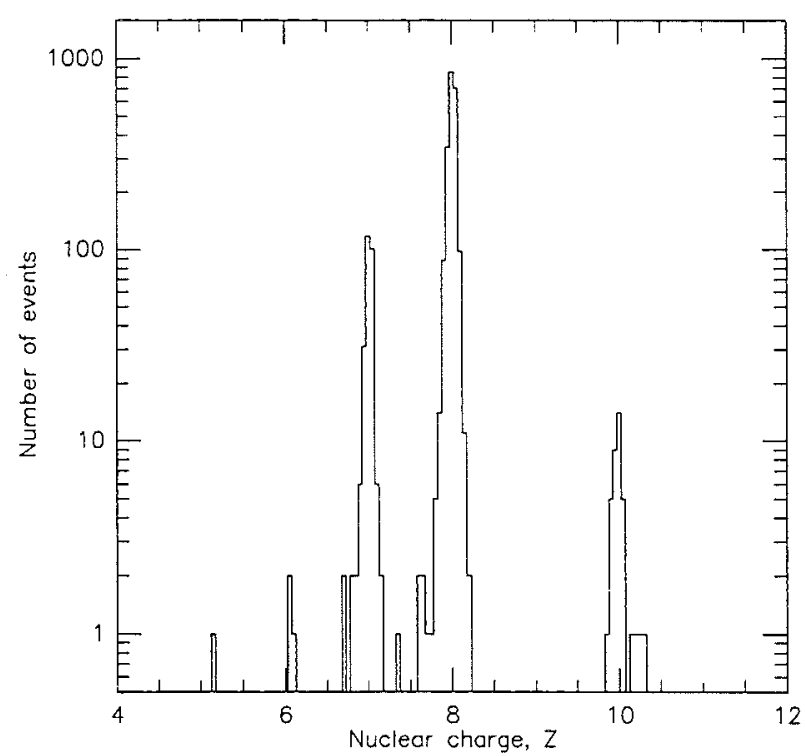

Figure 6. Composition of trapped ACRs with $>15 \mathrm{MeV} /$ nuc observed by the MAST sensor on SAMPEX from 7/92 to 2/94 (see Selesnick et al. [1995a]).

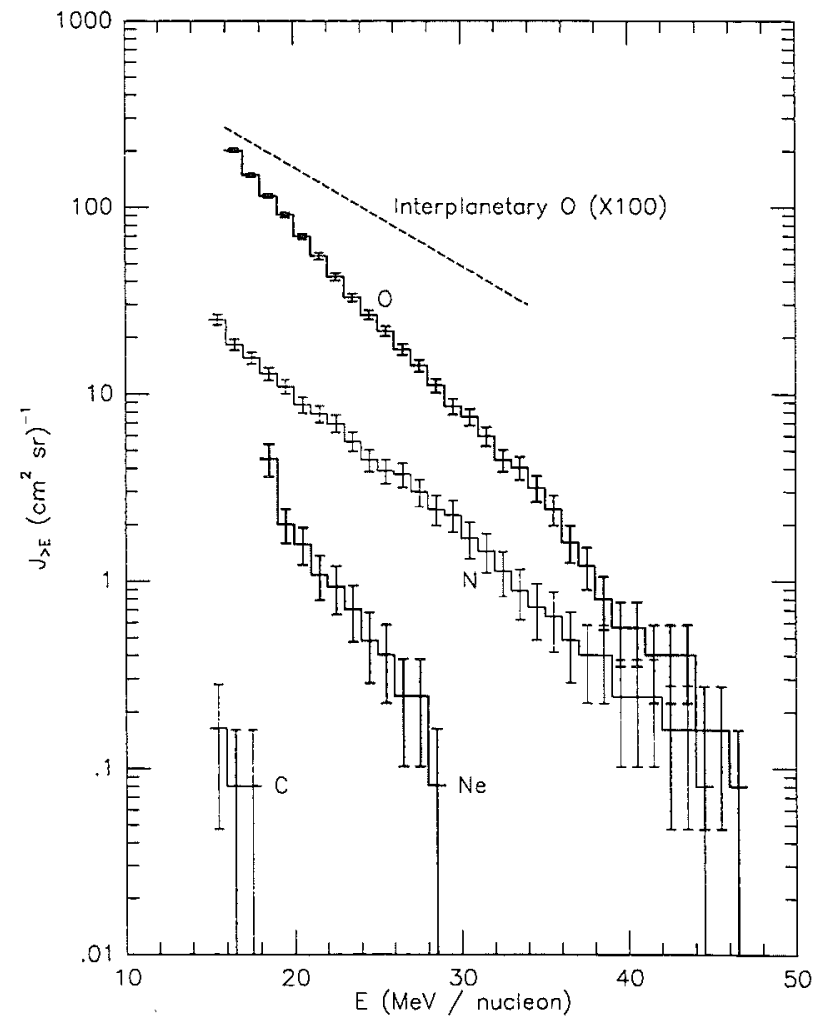

Figure 7. Integral energy spectra of trapped $\mathrm{C}, \mathrm{N}, \mathrm{O}$, and Ne fluences observed by SAMPEX from 7/92 to 2/94 (see Selesnick et al. [1995a]). The relative interplanetary oxygen spectrum observed by SAMPEX is also shown [Mewaldt et al., 1993]. 


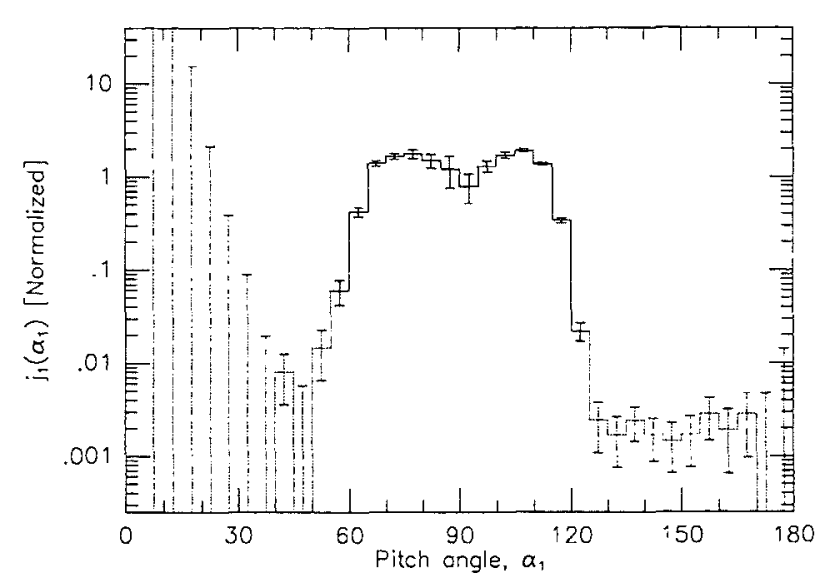

Figure 8. Normalized pitch angle distribution for trapped oxygen with $>16 \mathrm{MeV} /$ nuc. The pitch angles were transformed to a single value of $R=L \cos ^{2} \Lambda=1.3$, as described in Selesnick et al. [1995a].

has been used to measure the composition of a pure sample of ACRs and to extend measurements of the ACR energy spectrum $\sim 100 \mathrm{MeV} /$ nuc [Mewaldt et al., 1996].

The data in Figure 5 also illustrate Blake and Friesen's [1977] trapping mechanism. Low energy galactic cosmic rays or solar particles with charge states of $Q=A / 2$ do not have access to $L=2$, but singly-charged ACRs can penetrate down to the geomagnetic cutoff for $Q=1$. Once stripped, they can be trapped if they have the proper pitch angles, and if they undergo adiabatic motion, as characterized by the "adiabaticity" parameter

$$
\varepsilon=5.18 \times 10^{-5} \frac{A}{Q_{\mathrm{s}}} L^{2}[E(E+1863)]^{1 / 2} .
$$

Here $E$ is kinetic energy in $\mathrm{MeV} / \mathrm{nuc}, Q_{\mathrm{s}}$ is the charge state after stripping in the upper atmosphere, and particles are assumed to be stably trapped if $\varepsilon<\varepsilon_{0}$. Blake and Friesen [1977] originally suggested $\varepsilon_{0}=1 / 3$, in which case the trapped component in Figure 5 would extend out to $\Lambda=60^{\circ}$ $(L=4)$. Although the exact value of $\varepsilon_{0}$ is a subject of discussion, SAMPEX observations show that $\varepsilon_{0} \approx 0.1$ is a more appropriate value [Cumming s et al., 1993a; Tylka, 1994; Selesnick et al., 1995a]. Figure 5 illustrates that the trapping boundary for oxygen can be represented approximately by $\varepsilon Q=0.8$ (consistent with $\varepsilon_{0}=0.1$ and $Q_{\mathrm{s}}=8$ for oxygen).

The composition of trapped nuclei with $>15 \mathrm{MeV} /$ nuc at $L=2$ is shown in Figure 6 . In addition to $\mathrm{O}$ and $\mathrm{N}$, also detected by Grigorov et al. [1991] (see also Bobrovskaya et al. [1993]), a substantial amount of $\mathrm{Ne}$, and a small amount of $\mathrm{C}$ can be observed. There is also evidence for trapped $\mathrm{Ar}$ [Jonthal et al., 1993; Mazur et al., 1993]. From a comparison of these abundances with those of interplanetary ACRs (Table 1) it appears that ACR nitrogen may be less efficiently trapped than oxygen, while trapped ACR carbon is depleted by more than a factor of 40 (see discussion in Selesnick et al. [1995a]). This depletion of $\mathrm{C}$ is expected if $\varepsilon_{0}=0.1$ because $C$ with a rigidity just at the geomagnetic cutoff will

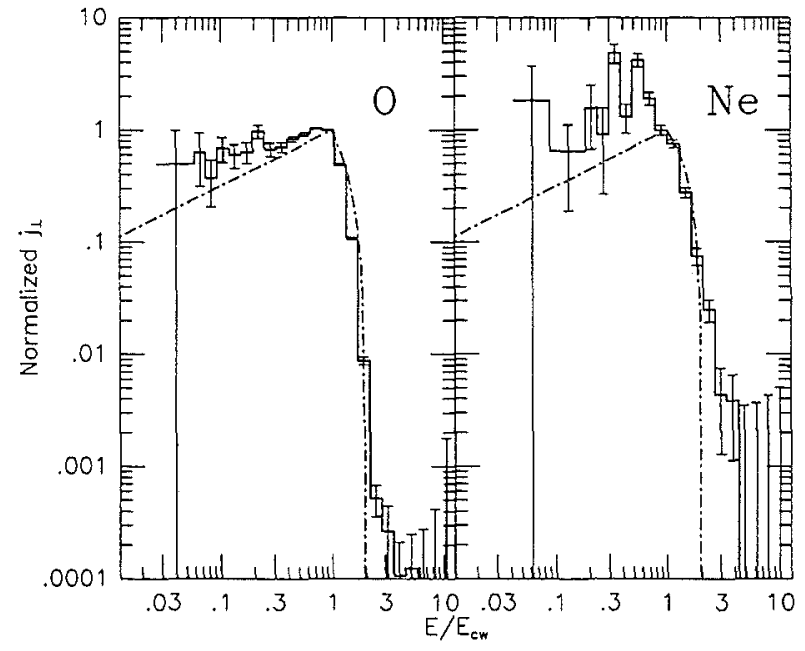

Figure 9. Spectra of trapped O and Ne based on data from the LICA, HILT, and MAST sensors on SAMPEX. The energy is normalized by the local western geomagnetic cutoff energy. Model spectra are shown by the dot-dash curves (see Selesnick et al. [1995b]). The differences at low energies may be due to approximations in the model or to possible systematic uncertainties in normalizing data from the three instruments.

have $\varepsilon Q>0.8$, even if $Q_{\mathrm{s}}=6$ after stripping, so apparently $C$ is difficult to trap. Similarly, it is not expected that ACR helium would be trapped at all by this mechanism.

The composition of trapped heavy ions in Table 1 is unlike that of any other suggested sources of magnetospheric ions [Cummings et al., 1993a]. The observed C/O ratio of $\sim$ 0.0004 is much less than that of solar energetic particles or the solar wind (where $\mathrm{C} / \mathrm{O} \sim 0.5$ ), the $\mathrm{Ne} / \mathrm{O}$ ratio of $\sim 0.04$ is much greater than that in the ionosphere (where $\mathrm{Ne} / \mathrm{O} \sim$ $\left.5 \times 10^{-4}\right)$. These and other sources may also contribute to the population of trapped heavy ions, especially at lower energies, where there have been a number of earlier observations of nuclei with $Z>2$ (see e.g., the review by Spjeldvik and Fritz [1983]). It is clear, however, that trapped ACRs have been the dominant source of high energy $(>10 \mathrm{MeV} /$ nuc) heavy ions with $Z>2$ observed in the radiation belts over the past decade.

The energy spectra of the trapped nuclei, shown in Figure 7 , are considerably softer than the corresponding interplanetary spectra. The spectrum for trapped 16 to $45 \mathrm{MeV} /$ nuc oxygen is approximately exponential with an e-folding energy of $\sim$ $4 \mathrm{MeV} /$ nuc. The e-folding energy for interplanetary oxygen is $\sim 8 \mathrm{MeV} /$ nuc. The trapped spectra also appear to soften with increasing atomic number. These differences are not understood, but may be due in part to the energy dependence of the stripping cross sections.

Assuming that the observed intensity $j$ is a separable function $j=U(E, L) V(\alpha) W(t)$ of energy/nuc $E$, pitch angle $\alpha$, and time $t$, Selesnick et al. [1995a] were able to determine the energy spectra, pitch angle distribution, and time variations of trapped $O$ in 1992 and 1993. Figure 8 shows that the pitch angle distribution of $>16 \mathrm{MeV} /$ nuc oxygen is peaked 
at $90^{\circ}$, with a broad, flat maximum. Selesnick et al. [1995a] argued that the nearly isotropic distribution observed outside the loss cone implies that electron stripping is a single-step process. In this case, both the source and loss rates of trapped ACRs would be proportional to the atmospheric density at the mirror point altitude, resulting in an isotropic pitch angle distribution at a given altitude.

This analysis approach has recently been extended to a broader energy interval ( $\sim 1$ to $50 \mathrm{MeV} / \mathrm{nuc})$ by including data from the LICA and HILT sensors on SAMPEX [Selesnick et al., 1995b]. An analysis of data from seven $L$-shells ranging from $L=1.66$ to $L=2.53$ shows that in each case the maximum intensity occurs at an energy corresponding to the western geomagnetic cutoff for singly-charged ions. Demonstration of this organization by geomagnetic cutoff is provided in Figure 9, in which data from all $L$-shells is plotted as a function of $E / E_{\mathrm{cw}}$, where $E_{\mathrm{cw}}$ is the western cutoff energy. Trapped particles with energies below the cutoff have apparently lost energy as they brushed against the upper atmosphere at their mirror points. The trapped spectra at energies above the geomagnetic cutoff are affected by the interplanetary source spectra, the energy dependence of the stripping cross sections, and the requirement for adiabatic motion [Selesnick et al., 1995ab]. Also shown in Figure 9 is the result of a simple model for trapped ACRs which is in reasonable agreement with the experimental data. Similar models have also been described by others [Blake, 1990; Tylka, 1994; Tylka et al., 1996].

\section{A SEMI-EMPIRICAL MODEL OF TRAPPED ANOMALOUS COSMIC RAYS}

The results described above determine the trapped ACR intensity at and below the SAMPEX orbit. Selesnick et al. [1995a] have also placed limits on the intensity of trapped ACRs above the SAMPEX orbit (see Figures 11 and 12 of their paper). A lower limit can be derived by considering the omnidirectional intensity due only to ions observed by SAMPEX, assuming that there are none with higher mirror points. A more reasonable estimate is obtained by assuming the intensity at $90^{\circ}$ pitch angles at points above the SAMPEX orbit is equal to the value observed at the orbit, as discussed above. Here the intensity generally increases with altitude on a given $L$-shell due to the narrowing of the loss cone. Note that the true intensity may differ from this if other loss processes are important, or if the fluxes have not attained a steady state because the trapping lifetimes are long compared to variations in the interplanetary source strength. It is planned to test this model with simultaneous SAMPEX/COSMOS observations at two altitudes during 1994-1996.

To estimate the intensity of trapped ACRs as a function of time we assume proportionality to the interplanetary source strength (Figure 2). Then the intensities presented in Selesnick et al. [1995a] can be scaled by the factor $F_{\mathrm{nm}}=(\text { Climax } / 3993)^{25}$ where "Climax" is the counting rate of the CLIMAX neutron monitor during the period of interest $(=3993$ for the SAMPEX 7/92 to $2 / 94$ period of Selesnick et al. [1995a] and $=4024$ for the $7 / 92$ to $2 / 95$ period of Selesnick et al. [1995b]). Over the first two and one-half years after the SAMPEX launch the flux of trapped ACRs has increased by a factor of $\sim 3$ to $\sim 4$, in reasonable cor- relation with the increase in the interplanetary fluxes as solar minimum approaches. For a typical solar minimum Climax rate of $\sim 4250$ the expected trapped ACR fluxes are $\sim 5$ times higher than in Selesnick et al. [1995a]. The ratio of the trapped oxygen to trapped proton fluxes at $15 \mathrm{MeV} /$ nuc is $\sim 10^{-6}$.

\section{SUMMARY}

All observations to date of trapped ACRs confirm that the basic mechanism proposed by Blake and Friesen [1977], and amplified by others, is responsible for the belt of trapped ACRs observed at $L=2$. The mechanism appears to be reasonably efficient for $\mathrm{N}, \mathrm{O}, \mathrm{Ne}$, and possibly $\mathrm{Ar}$, but $\mathrm{C}$ is apparently trapped very inefficiently, and ACR He is not trapped at all. Although simple models of the trapping mechanism are able to account for the observations, there remain questions as to the relevant stripping cross sections, and the associated trapping efficiencies and lifetimes. The Cosmos observations suggest that trapped ACRs vary in intensity by more than two orders of magnitude over the solar cycle, in proportion to the interplanetary ACR intensity. The trapped intensity has increased by a factor of $\sim 3$ to 4 from mid-1992 to early 1995, and may increase by another factor of 2 or 3 by the time of solar minimum.

Acknowledgements. This work was supported by NASA under contract NAS5-30704 and grant NAGW-1919. We appreciate contributions to this work by our SAMPEX colleagues B. Blake, A. Cummings, B. Klecker, R. Leske, G. Mason, J, Mazur, E. Stone, and T. von Rosenvinge. We thank B. Klecker, R. Pyle, and J. Simpson for the use of unpublished data in Figure 2.

\section{NOTE ADDED IN PROOF}

Since the submission of this paper new measurements by SAMPEX [Mewaldt et al., Ap. J. Letters, in press, 1996] have shown that the vast majority of interplanetary ACRs with $>30 \mathrm{MeV} /$ nuc are multiply-charged, with charge states of $Q=2, Q=3$, and probably higher. This contrasts to energies of $\sim 10 \mathrm{MeV} /$ nuc, where most ACRs are singly charged. As pointed out by Mewaldt et al. [1996], the dominance of multiply-charged ACRs at high energy can explain why trapped ACRs have a steeper energy spectrum than interplanetary ACRs (Figure 7), and why the trapped ACR spectrum extends only to $\sim 45 \mathrm{MeV} /$ nuc, much less than the interplanetary spectrum (see Figure 5). To be trapped by the Blake and Friesen [1977] mechanism interplanetary ions must have access to invariant latitudes below the $\varepsilon Q=0.8$ line in Figure 5. Since only singly-charged oxygen can reach this region, trapped ACR oxygen is derived from only $\mathrm{O}^{+1}$, and not from multiply-charged ACR oxygen that dominates at higher energy.

\section{REFERENCES}

Baker, D.N., G.M. Mason, O. Figueroa, G. Colon, J.G. Watzin and R.M. Aleman, An overview of the Solar, Anomalous, and Magnetospheric Particle Explorer (SAMPEX) mission, IEEE Trans. Geosci. Remote Sensing, 31, 531-541, 1993.

Blake, J.B., Geomagnetically trapped heavy ions from anomalous 
cosmic rays, Proc. $21^{\text {st }}$ Internat. Cosmic Ray Conf. (Adelaide), 7, 30-33, 1990.

Blake, J.B. and L.M. Friesen, A technique to determine the charge state of the anomalous low-energy cosmic rays, Proc. $15^{\text {th }}$ Internat. Cosmic Ray Conf. (Plovdiv), 2, 341-346, 1977.

Bobrovskaya, V., N.L. Grigorov, M.A. Kondratyeva, M.I. Panasyuk, Ch.A. Tretyakova, D.A. Zuravlev, J.H. Adams, Jr. and A.J. Tylka, Cosmos observations of anomalous cosmic ray $\mathrm{N}$ and $\mathrm{Ne}$ in the inner magnetosphere, Proc. $23^{\text {rd }}$ Internat. Cosmic Ray Conf. (Calgary), 3, 432-435, 1993

Chan, J.H. and P.B. Price, Composition and energy spectra of heavy nuclei of unknown origin detected on Skylab, Astrophys. J., 375 , L539-L542, 1975.

Cook, W.R., A.C. Cummings, J.R. Cummings, T.L. Garrard, B. Kecman, R.A. Mewaldt, R.S. Selesnick, E.C. Stone and T.T. von Rosenvinge, MAST: A mass spectrometer telescope for studies of the isotopic composition of solar, anomalous, and galactic cosmic ray nuclei, IEEE Trans. Geosci. Remote Sensing, 31, 557-564, 1993.

Cummings, A.C. and E.C. Stone, Elemental composition of the anomalous cosmic ray component, Proc. $24^{\text {th }}$ Internat. Cosmic Ray Conf. (Rome), 4, 497-500, 1995.

Cummings, J.R., A.C. Cummings, R.A. Mewaldt, R.S. Selesnick, E.C. Stone and T.T. von Rosenvinge, New evidence for geomagnetically trapped anomalous cosmic rays, Geophys. Res. Lett., 20 , 2003-2006, 1993a.

Cummings, J.R., A.C. Cummings, R.A. Mewaidt, R.S. Selesnick, E.C. Stone, T.T. von Rosenvinge and J.B. Blake, SAMPEX measurements of heavy ions trapped in the magnetosphere, IEEE Trans. Nucl. Sci., 40, 1459-1462, 1993 b.

Cummings, J.R., A.C. Cummings, R.A. Mewaldt, R.S. Selesnick, E.C. Stone and T.T. von Rosenvinge, SAMPEX observations of geomagnetically trapped anomalous cosmic rays, in Proceedings of the $23^{\text {rd }}$ Internat. Cosmic Ray Conf., Invited, Rapporteur and Highlight Papers, edited by D.A. Leahy, R.B. Hicks, and D. Venkatesan, pp. 475-482, World Scientific, Singapore, 1994.

Fisk, L.A., B. Kozlovsky and R. Ramaty, An interpretation of the observed oxygen and nitrogen enhancements in low-energy cosmic rays, Astrophys. J., 190, L35-L38, 1974.

Geiss J., G. Gloeckler, U. Mall, R. von Steiger, A.B. Galvin and K.W. Ogilvie, Interstellar oxygen, nitrogen, and neon in the heliosphere, Astron. Astrophys., 282, 924-933, 1994.

Grigorov, N., M.A. Kondratyeva, M.I. Panasyuk, Ch.A. Tretyakova, J.H. Adams, Jr, J.B. Blake, M. Schulz, R.A. Mewaldt and A.J. Tylka, Evidence for anomalous cosmic ray oxygen ions in the inner magnetosphere, Geophys. Res. Lett., 18, 1959-1962, 1991.

Jokipii, J.R., The anomalous component of cosmic rays, in Physics of the Outer Heliosphere, S. Grzedzielski and D.E. Page, Pergamon Press, 1990

Klecker, B., The anomalous component of cosmic rays in the 3-D heliosphere, Space Science Reviews, 72, 419-430, 1995.

Klecker, B., M.C. McNab, J.B. Blake, D. Hovestadt, H. Kastle, D.C. Hamilton, M.D. Looper, G.M. Mason, J.E. Mazur and M. Scholer, Charge state of anomalous cosmic ray nitrogen, oxygen, and neon: SAMPEX observations, Astrophys. J., 442, L69-L72, 1995.

Mazur, J.E. and G.M. Mason, Observations of low energy trapped anomalous cosmic rays using SAMPEX, Trans. Am. Geophys. U., 76, S 237, 1995.
Mewaldt, R.A., A.C. Cummings, J.R. Cummings, E.C. Stone, B. Klecker, D. Hovestadt, M. Scholer, G.M. Mason, J.E. Mazur, D.C. Hamilton, T.T. von Rosenvinge and J.B. Blake, The return of the anomalous cosmic rays to $1 \mathrm{AU}$ in 1992, Geophys. Res. Lett., 20, 2263-2266, 1993.

Mewaldt, R.A., J.R. Cummings, R.A. Leske, R.S. Selesnick, E.C. Stone and T.T. von Rosenvinge, A study of the composition and energy spectra of anomalous cosmic rays using the geomagnetic field, Geophys. Res. Letters, in press, 1996.

Mewaldt, R.A., A.C. Cummings and E.C. Stone, The anomalous cosmic rays-Interstellar interlopers in the heliosphere and the magnetosphere, EOS Trans. Am. Geophys. Un., 85, 185 and 193 , 1994.

Pesses, M.E., J.R. Jokipii and D. Eichler, Cosmic ray drift, shock wave acceleration, and the anomalous component of cosmic rays, Astrophys. J., 246, L85-L88, 1981.

Selesnick, R.S., A.C. Cummings, J.R. Cummings, R.A. Mewaldt, E.C. Stone and T.T. von Rosenvinge, Geomagnetically trapped anomalous cosmic rays, J. Geophys. Res., 100,9503-9509, 1995a.

Selesnick, R.S., R.A. Mewaldt, E.C. Stone, G.M. Mason, J.E. Mazur, J.B. Blake, M.D. Looper, B. Klecker and D. Hovestadt, Observations of geomagnetically trapped anomalous cosmic rays, Proc. $24^{\text {th }}$ Internat. Cosmic Ray Conf. (Rome) 4, 1013-1017, $1995 b$.

Simpson, J.A.; The anomalous nuclear component in the threedimensional heliosphere, Adv. Space Res., 16, 135-149, 1995.

Spjeldvik, W.N., and T.A. Fritz, Experimental determination of geomagnetically trapped energetic heavy ion fluxes, Energetic Ion Composition in Earth's Magnetosphere, R.G. Johnson, ed., Terra Scientific, Tokyo, 1983.

Tylka, A.J., Theoretical modeling and interpretation of trapped anomalous cosmic rays, in Proceedings of the $23^{\text {rd }}$ International Cosmic Ray Conference, Invited, Rapporteur and Highlight $\mathrm{Pa}$ pers, edited by D.A. Leahy, R.B. Hicks and D. Venkatesan, pp. 475-482, World Scientific, Singapore, 1994.

Tylka, A.J., P.R. Boberg and J.H. Adams, Jr., LET spectra of trapped anomalous cosmic rays in low-earth orbit, Adv. Space Res., 17, $47-51,1996$.

R.A. Mewaldt, R.S. Selesnick and J.R. Cummings, California Institute of Technology, Pasadena, CA 91125, USA.

\section{DISCUSSION}

Q: A.L. Vampola. Why do you assume that the anomalous cosmic rays don't peak low on the field line? The data seems to show this and the maximum generation is down there.

A: R.A. Mewaldt. The lifetime of the particles scales with the source rate, so we expect an isotropic distribution initially. We will compare this with COSMOS data.

Q: G. Ginet. What is the ratio of the equatorial omnidirectional flux of the anomalous radiation belt to the standard electron and proton belts?

A: R.A. Mewaldt. I do not have the numbers right in front of me but I believe the ratio is at most $10^{-4}$. 
